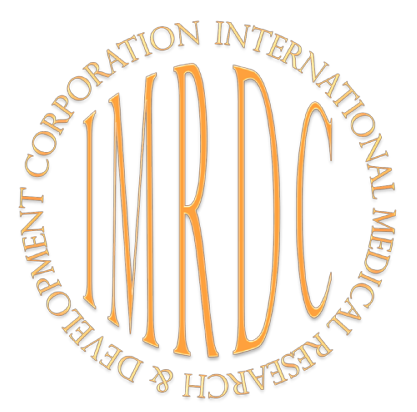

\title{
Prevalence of Tick-Borne Pathogens in Hard Ticks That Attacked Human Hosts in Eastern Siberia
}

\author{
Maxim A. Khasnatinov, PhD*; Galina A. Danchinova, PhD, ScD; \\ Alexander V. Liapunov, PhD; Ellina L. Manzarova; Irina V. Petrova; \\ Natalie A. Liapunova (Bolotova); Innokentiy S. Solovarov \\ Scientific Center for Family Health and Human Reproduction Problems \\ Irkutsk, the Russian Federation
}

\begin{abstract}
The aim of this study was to evaluate the risk of tick-borne infections in humans. The prevalence of 4 tick-borne pathogens was studied in the population of Ixodid ticks attacking human hosts in Irkutsk city and neighbouring territories from 2007 to 2017.

Methods and Results: In total, 46,357 tick specimens detached from bitten people were analyzed. The antigen of tick-borne encephalitis virus (TBEV) was detected in each tick individually by ELISA assay using a commercial kit for the envelope protein E of TBEV. Total RNA and DNA were extracted from ticks using a RiboPrep kit. Reverse transcription was performed using a Reverta-L kit and RNA\DNA of TBEV; B. burgdorferi sensu lato, A. phagocytophylum and Ehrlichia muris $\backslash$. chaffeensis were detected using a real-time multiplex PCR kit. In total, during 8 years of observations, I. persulcatus caused approximately $86 \%$ of bites, Dermacentor $s p .13 .95 \%$, and H. concinna $0.05 \%$. The most prevalent tick-borne pathogen in I. persulcatus ticks was Lyme disease agent $B$. burgdorferi sensu lato, which was detected in $12 \pm 6.5 \%$ of specimens annually. A. phagocytophilum and Ehrlichia sp. were detected in $7.8 \pm 2.7 \%$ and $4.6 \pm 1.5 \%$ of specimens, respectively. TBEV was present in $1 \pm 0.7 \%$ of I. persulcatus.

Conclusion: I. persulcatus remains the most important vector of tick-borne diseases to humans in Eastern Siberia. D. nuttalli and $D$. silvarum are much less aggressive to humans and are less infected with major tick-borne pathogens. H. concinna does not play any significant role as a disease vector. However, a rigorous analysis of TBEV spread in the Dermacentor $s p$. population is necessary. (International Journal of Biomedicine. 2017;7(4):307-309.)
\end{abstract}

Key Words: Tick-borne encephalitis virus $\bullet$ Borrelia burgdorferi sensu lato $\bullet$ Anaplasma phagocytophilum $\bullet$ Erlichia muris

\section{Introduction}

Ixodid ticks are the vectors of several dangerous human pathogens. The most important of them are tick-borne encephalitis virus (TBEV) and Borrelia burgdorferi sensu lato (Spirochaetaseae). TBEV causes about 3,000 cases of tickborne encephalitis (TBE) in Europe and up to 10,000 cases in the Russian Federation annually. ${ }^{(1,2)}$ The agent of Lyme disease, B. burgdorferi sensu lato, is spread worldwide and causes approximately 85,500 cases of disease annually, including up to 9,000 cases per year in the Russian Federation. ${ }^{(1,3)}$ Several "emerging" tick-borne diseases (TBDs) have been described

*Corresponding author: Maxim A. Khasnatinov, PhD. Scientific Center for Family Health and Human Reproduction Problems. Irkutsk, Russia.E-mail: khasnatinov@gmail.com in recent decades, including human granulocytic anaplasmosis and human monocytic ehrlichiosis caused by Anaplasma phagocytophilum and Ehrlichia chaffeensis, respectively. ${ }^{(4)}$

In the Irkutsk region (Eastern Siberia, Russia), TBDs pose serious threats to the health of local residents and visitors. To reduce the risk of infection, the Centre for Diagnosis and Prevention of Tick-Borne Diseases (hereinafter, the Centre) was established at our institution. According to sanitary rule SP3.1.3.2352-08, each tick is routinely tested for infection with TBEV and B. burgdorferi sensu lato, and if the pathogen is detected in the tick, the patient receives the treatment with anti-TBEV immunoglobulins and/or antibiotics to prevent the disease according to modern Russian healthcare regulations. ${ }^{(5)}$ Approximately 7,000 bite victims attend the Centre after tick bites annually, and more than half of them deliver the causative tick specimen. ${ }^{(6)}$ In this study, we evaluated the prevalence of tick-borne pathogens in the population of Ixodid ticks that 
attacked humans in Irkutsk city and neighbouring territories during 11 consecutive seasons of tick activity between 2007 and 2017.

\section{Materials and Methods}

\section{$\underline{\text { Ticks }}$}

The study was performed in Irkutsk city and neighbouring territories within a range of about $100 \mathrm{~km}$. This area is situated in Eastern Siberia with landscapes formed by typical taiga forests and harsh continental (borderline subarctic) climate. Ticks were delivered to the Centre by bitten people between March 25, 2007, and October 17, 2017. Each patient was interviewed to collect the following information: the time, geographic location and circumstances of the tick attack; the age, gender and occupation of the patient; the history of vaccination against TBE; the history of tick bites and TBDs in the past; and, finally, the current state of the patient's health. The study was approved by the Ethics Committee of Scientific Center for Family Health and Human Reproduction Problems. Written informed consent was obtained from each patient.

\section{Tick species identification}

Each tick was washed twice in $70 \%$ ethanol and once in distilled water, then dried on filter paper. Its life stage and species were identified, and then it was immediately dissected for pathogen detection. The developmental stage and identification of tick species was done using key guides to tick fauna of Russia and adjacent countries. ${ }^{(7-9)}$ Due to time restrictions, the $D$. nuttalli and D. silvarum tick species were identified only to the genus level and are hereafter designated as Dermacentor sp.

\section{Detection of tick-borne pathogens}

The agents of Lyme disease were detected by light microscopy according to the standard procedure. ${ }^{(10)}$ The antigen of TBEV was detected in each tick individually by ELISA assay using a commercial kit for the envelope protein E of TBEV (Vector-Best, Novosibirsk). Total RNA and DNA were extracted from ticks using a RiboPrep kit (AmpliSens, Moscow). Reverse transcription was performed using a Reverta-L kit (Amplisens, Moscow) and RNA DNA of TBEV; B. burgdorferi sensu lato, A. phagocytophylum and Ehrlichia muris $\backslash E$. chaffeensis (hereinafter, Ehrlichia sp.) were detected using a real-time multiplex PCR kit "AmpliSens ${ }^{\circledR}$ TBEV, B.burgdorferi sensu lato, A. phagocytophilum, E. chaffeensis/E. muris - FL" (Amplisens, Moscow).

Results were presented as mean values, where appropriate. To evaluate the variability of the data, we estimated the standard deviations of a mean. The long-term trends of the dynamics of tick attack rates were evaluated using linear approximation. Calculations were performed using the MSOffice EXCEL software package.

\section{Results}

In total, 46,357 tick specimens detached from bitten people were analyzed. The most abundant species was $I$. persulcatus with a mean incidence of 3,620 4473 attacks per year. Two closely related Dermacentor species provided a mean incidence of $591 \pm 133$ attacks per year, whereas another endemic species, $H$. concinna, appeared to be less aggressive to humans with only $2.3 \pm 1.5$ attacks per year. In total, during 11 years of observations, I. persulcatus caused approximately $86 \%$ of bites, Dermacentor sp. $13.95 \%$, and H. concinna $0.05 \%$. The 11-year dynamics of tick attachment to human hosts is present in Fig.1. The trend toward an increase of the attack rate was observed for I. persulcatus, though the reliability of approximation is low $\left(\mathrm{R}^{2}=0.4309\right)$.

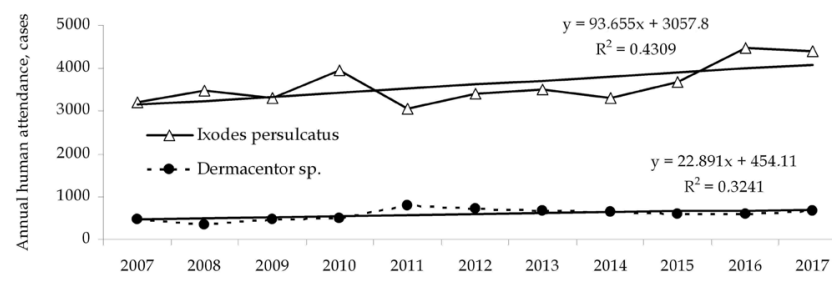

Fig. 1. The 11-year dynamics (2007-2014) of tick attachment to human hosts.

The most prevalent tick-borne pathogen in I. persulcatus ticks was Lyme disease agent $B$. burgdorferi sensu lato, which was detected in $12 \pm 6.5 \%$ of specimens annually. $A$. phagocytophilum and Ehrlichia sp. were detected in $7.8 \pm 2.7 \%$ and $4.6 \pm 1.5 \%$ of specimens, respectively. TBEV was present in $1 \pm 0.7 \%$ of $I$. persulcatus. In Dermacentor sp., the prevalence of every infection was below $5 \%$, with the highest rate for $A$. phagocytophilum $(4.2 \pm 3.7 \%)$. The 11-year dynamics indicate some increase of infection rates of TBEV and B. burgdorferi sensu lato in I. persulcatus (Fig. 2A, B); however, the changes in Dermaventor $s p$. are not obvious. In 2014, the prevalence of A. phagocytophilum and Ehrlichia sp. was unusually high among both $I$. persulcatus and among Dermacetor $s p$, but it has decreased during last three years (Fig. 2 C, D). None of the infections were detected in $H$. concinna ticks.
A

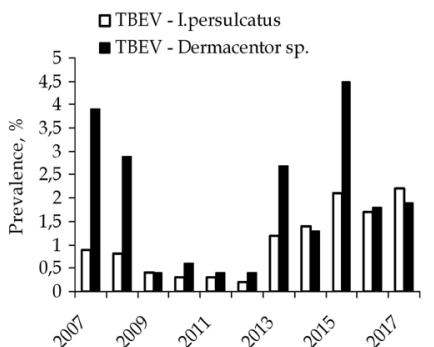

C

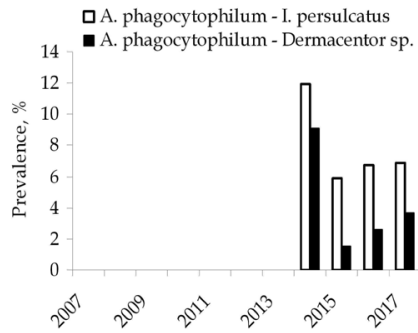

B

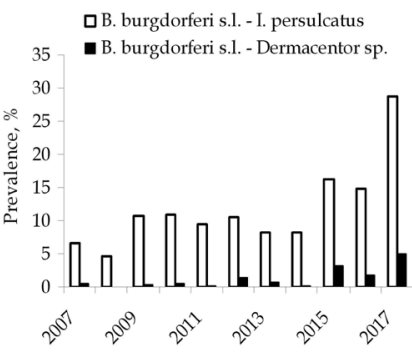

$\mathrm{D}$

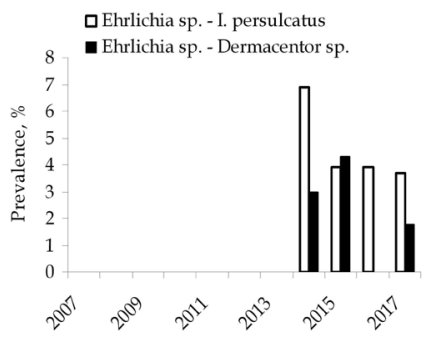

Fig. 2. Annual prevalence of infections in Ixodes persulcatus and Dermacentor sp. for TBEV (A), B. burgdorferi sensu lato (B), A. phagocytophilum (C), and Ehrlichia sp. (D) 


\section{Discussion}

As usual, I. persulcatus remains the most important vector of TBDs to humans in Eastern Siberia. D. nuttalli and $D$. silvarum are much less aggressive to humans, whereas $H$. concinna does not play any significant role as a disease vector, though its abundance in Eastern Siberian landscapes may reach 14 ticks per one kilometre. ${ }^{(11)}$ This is probably because of the mosaic distribution of $H$. concinna caused by the relatively rare natural conditions suitable for these ticks, such as wet deciduous forests and shrub meadows.

The dynamics of TBEV prevalence indicate a trend toward a significant increase in the infection rate during 2014 2017. This increase was more characteristic of I. persulcatus, whereas the data for Dermacentor sp. ticks were not as clear and exhibited high variation between consecutive years. Unpredictable variations of data in Dermacentor $s p$. ticks, in combination with a relatively small sample volume, indicate the need for rigorous analysis of TBEV spread among these ticks. For the Lyme disease agent, a similar increase was observable in the I. persulcatus population; however, this pathogen is only sporadically present in Dermacentor $s p$. ticks.

Thus, about $86 \%$ of ticks were identified as Ixodes persulcatus; the Dermacentor sp. caused about $14 \%$ of bites, whereas Haemaphysalis concinna caused 1-5 bites per year. The mean prevalence of infection in $I$. persulcatus equalled $12 \%, 7.8 \%, 4.6 \%$ and $1 \%$ for $B$. burgdorferi sensu lato, $A$. phagocytophylum, Ehrlichia sp., and TBEV, respectively.

I. persulcatus remains the most important vector of tickborne diseases to humans in Eastern Siberia, with the highest attack rate and with about $25 \%$ of ticks being infected with at least one of four pathogens. D. nuttalli and D. silvarum are much less aggressive to humans and are less infected with major tick-borne pathogens. $H$. concinna does not play any significant role as a disease vector. However, a rigorous analysis of TBEV spread in the Dermacentor $s p$. population is necessary.

\section{Competing interests} interests.

\section{Acknowledgments}

The study was partially supported by the Russian Fund for Basic Research (Project No.15-47-04348).

\section{References}

1. Korenberg E, Likhacheva T. Analysis of the long-term dynamics of tick-borne encephalitis (TBE) and ixodid tickborne borrelioses (ITBB) morbidity in Russia. Int J Med Microbiol. 2006;296(Suppl 40):54-8.

2. Donoso Mantke O, Schädler R, Niedrig M. A survey on cases of tick-borne encephalitis in European countries. Euro Surveill. 2008;13(17). pii:18848.

3. Hubalek Z. Epidemiology of Lyme borreliosis. Curr Probl Dermatol. 2009;37:31-50. doi: 10.1159/000213069.

4. Ismail N, Bloch KC, McBride JW. Human ehrlichiosis and anaplasmosis. Clin Lab Med. 2010;30(1):261-92. doi: 10.1016/j.cll.2009.10.004.

5. Prevention of tick-borne viral encephalitis. Sanitary rules SP3.1.3.2352-08. Moscow: Federal Service for Supervision of Consumer Rights Protection and Human Welfare; 2008. [in Russian].

6. Khasnatinov MA, Lyapunov AV, Danchinova GA, Chaporgina EA, Arbatskaya EV, Tunik TV, Petrova IV. Tickborne encephalitis: the incidence and prevention of preclinical infection among victims bitten by Ixodid ticks. Epidemiol Inf Dis Current Items. 2012;5:19-24. [Article in Russian].

7. Filippova NA. Ixodid ticks of the subfamily Ixodinae. Fauna of Russia and adjacent countries. Arachnida. Leningrad: Nauka; 1977, 4(4). [in Russian].

8. Serdjukova GV. Ixodid ticks of USSR fauna. M., L.: Publishing house of the Academy of Sciences of USSR; 1956. [in Russian].

9. Filippova NA. Ixodid ticks of the subfamily Amblyomminae., "Fauna of Russia and Adjacent Countries. Arachnida." St. Petersburg: Nauka; 1997, 4(5). [in Russian]. 10. Methodical instructions on epidemiology, diagnosis, clinical and prevention of Lyme disease. Edited by: Korenberg EI. Moscow: Ministry o HealthCare of USSR;1991, 15(6/12). [in Russian].

11. Vershinin EK, Mel'nikova OV, Morozov IM. Haemaphysalis ticks in the southern part of Pribaikalie. Bull Irk State Univer, Series «Biology, Ecology». 2014;8:92-5. [Article in Russian]. 